\title{
870 TARGETING GCN2 KINASE-DRIVEN STRESS RESPONSE INACTIVATION TO RESTORE TUMOR IMMUNITY IN METASTATIC TRIPLE NEGATIVE BREAST CANCER
}

${ }^{1}$ Hariprasad Vankayalapati, 'Kyle Medley, 'Zhaoliang Li*, 'Dongqing Yan, ${ }^{1}$ David Bearss,

${ }^{1}$ Alana Welm. ' Huntsman Cancer Institute, Salt Lake City, UT, USA; ${ }^{2}$ University of Utah, Salt Lake City, UT, USA

Background Patients with PD-L1-positive metastatic triple-negative breast cancers (mTNBC) who have been treated with atezolizumab+nab-paclitaxel had a clinically meaningful overall survival extension of 9.5 months compared to nab-paclitaxel alone, although overall survival in overall population was not statistically significant. Unlike many other cancers, immunotherapy for breast cancer has had limited success, due to the fact that there are very few $\mathrm{T}$ cells in the tumor microenvironment of mTNBC patients. Identifying ways to boost immunotherapy responses could change the paradigm of mTNBC, a disease still difficult to treat. The highly proliferative nature of tumor cells, along with infiltration of myeloid cells into the tumors, leads to depletion of nutrients such as functional/natural amino acids. This metabolically stressful milieu causes activation of nutrient stress pathways, autophagy, and repressed immune responses. A key meditator of this nutrient stress pathway is a cytoplasmic Ser/Thr protein kinase called General Control Nonderepressible 2 (GCN2), also called EIF2AK4. GCN2 switches on following reduction of amino acids, and its activity results in $\mathrm{T}$ cell inactivation, $\mathrm{T}$ cell death, regulatory $\mathrm{T}$ cell expansion, and the potentiation of myeloid-derived suppressor cells (MDSCs).

Methods We have developed and synthesized a series of novel small molecule immunotherapeutic agents that reversibly bind to GCN2 kinase, competitively block the ATP site, and elicit pharmacological responses in immune cells and in breast cancer cells.

Results GCN2 cell-free kinase binding, kinome selectivity, pGCN2, pEIF2 $\alpha$, ATF-4 phosphorylation inhibition assays were performed. We confirmed on-target efficacy and tested the potency of our lead GCN2 inhibitor HCI-1046. HCI1046 demonstrated potent activity, with an IC50 of $36 \mathrm{nM}$ in inhibiting GCN2 kinase and exhibited cellular efficacy with an IC50 of 0.1 to $1.0 \mu \mathrm{M}$ range. Our preliminary results support the hypothesis that the inhibition of GCN2 reinstates antitumor immunity and blocks tumor progression in breast cancer models. In vivo PK studies of HCI-1046 in rodents showed excellent PK properties; 55\% oral bioavailability, low clearance, and $>5$ hour half-life.

Conclusions Thus, HCI-1046 is nominated as a pre-clinical agent. Additional data regarding evaluation of the effects of HCI-1046 on the MDSC-suppressive function on T cells using ELISpot assays with breast cancer patient samples, and mouse model efficacy studies will be discussed.

\section{REFERENCES}

1. Ekiz HA, Lai SA, Gundlapalli H, Haroun F, Williams MA, Welm AL. Inhibition of RON kinase potentiates anti-CTLA-4 immunotherapy to shrink breast tumors and prevent metastatic outgrowth. Oncoimmunology 2018;7(9):e1480286.

2. Toogood PL. Small molecule immuno-oncology therapeutic agents. Bioorg Med Chem Lett 2018:28(3):319-329.

3. Ravindran R, Loebbermann J, Nakaya HI, Khan N, Ma H, Gama L, Machiah DK, Lawson B, Hakimpour P, Wang YC, Li S, Sharma P, Kaufman RJ, Martinez J, Pulendran B. The amino acid sensor GCN2 controls gut inflammation by inhibiting inflammasome activation. Nature 2016;531(7595):523-527.

4. Brazeau JF, Rosse G. Triazolo[4,5-d]pyrimidine derivatives as inhibitors of GCN2. ACS Med Chem Lett 2014;5(4):282-3. 